\title{
Bang-Bang Attitude Control During East-West Station Keeping For Geostationary Satellite
}

\author{
Elhassen BENFRIHA ${ }^{(1)^{*}}$, Jalel Eddine BENMANSOUR ${ }^{(1)}$, Abdellatif Bellar ${ }^{(1)}$ \\ ${ }^{(1)}$ Département de recherche en mécanique spatiale, Centre de Développement des Satellites, \\ Oran, ALGERIA. \\ "Corresponding author: hassen_ing@yahoo.fr
}

\begin{abstract}
This paper investigates the East-West station keeping strategy of geostationary satellites which must be performed to correct the drift caused by natural perturbation forces. The attitude control requirements as well as the pointing accuracy must be maintained during the station-keeping maneuver in order to assure the mission services. The propulsion system (chemical thrusters) is used to control both the orbit control (station keeping) and the attitude control where the ON/OFF thrusters pulses are managed using Bang-Bang controller with dead-band technique. Simulation results can clearly illustrate the feasibility and the effectiveness of the proposed control strategy.
\end{abstract}

Keywords: Geostationary satellite, Thruster attitude control, Station keeping, Bang-Bang, Dead-band

\section{INTRODUCTION}

The Communication satellites in geostationary orbit are subjected to several natural perturbations, which can affect the required position of satellite. For this purpose, station keeping (SK) maneuvers are periodically performed using the propulsion system in order to correct the orbit drift and maintain the satellite in its control box [1], [2].

The north-south station keeping (NSSK) is performed to correct the inclination drift in latitude due to luni-solar perturbations, whilst, the east-west station keeping (EWSK) is performed to correct the semi major axis/ eccentricity drift in longitude due to earth gravitational force and solar radiation pressure perturbations [3].

Otherwise, the attitude control as well as the satellite pointing should be assured during all satellite mission, momentum/reaction wheels are usually used for applying torques so that the satellite keeps its required orientation. However, in order to maintain the satellite in its control box the thrusters are used to compensate the orbit drift and modify the changes in the osculating orbit parameters, for this purpose, thruster control strategy is proposed by combining the attitude control and orbit control using only thrusters as actuators [4],[5].

Chemical thrusters generally operate in ON/OFF mode which impose severe restrictions represented in limiting the control authority. Furthermore, the performance of these actuators as well as their lifetime can be affected by the thruster switching frequency.

In order to reject disturbances and to ensure minimum thruster firing time, The used ON/ OFF thrusters for spacecraft pointing and stabilization requires a switching controller as a kind of pulse modulation [6], [7], [8].

The Bang-Bang controller is widely proposed in thruster attitude control in various cases such as in the case of single axis [9] and the case of small angle maneuvers [10],

However, other techniques must be combined to Bang-Bang controller in order to minimize the switching frequency and the fuel consumption, that permits to achieve best efficiency.

In the present paper, Bang-Bang controller with dead-band technique has been applied to manage the thruster firing control in order to insure both the attitude control and the orbit control (station keeping) simultaneously.

Finally, the obtained results can demonstrate the feasibility and the effectiveness of the proposed methods.

\section{SPACECRAFT DESCRIPTION}

Communication satellites in geostationary orbit are generally composed of the following subsystems [11]: 
- Attitude and Orbit Control System (AOCS)

- Electrical Power Subsystem (EPS)

- Unified Propulsion Subsystem (UPS)

- Telemetry, Commanding and Ranging subsystem

- Repeater Subsystem

- Thermal Control Subsystem (TCS)

\subsection{Attitude and orbit control subsystem:}

The AOCS is composed of sets of sensors and actuators [11].

The used sensors are:

- Sun sensors: 2 redundant sets each composed of 4 sensor heads.

- Earth sensors: two units for only roll and pitch measurement.

- Rate integrated gyroscope assembly: two redundant sets of gyros each gyro is composed of three gyroscopes.

The satellite actuators are:

- Momentum wheel assembly (MWA)

- $\quad 10 \mathrm{~N}$ Thrusters: Twelve $10 \mathrm{~N}$ thrusters are used to correct and maintain the satellite attitude and orbit, redundant branches of thrusters are formed each composed of six thrusters where branch $A$ is the nominal one and branch $B$ is the redundant one.

The thruster's configuration is presented in the figure below:

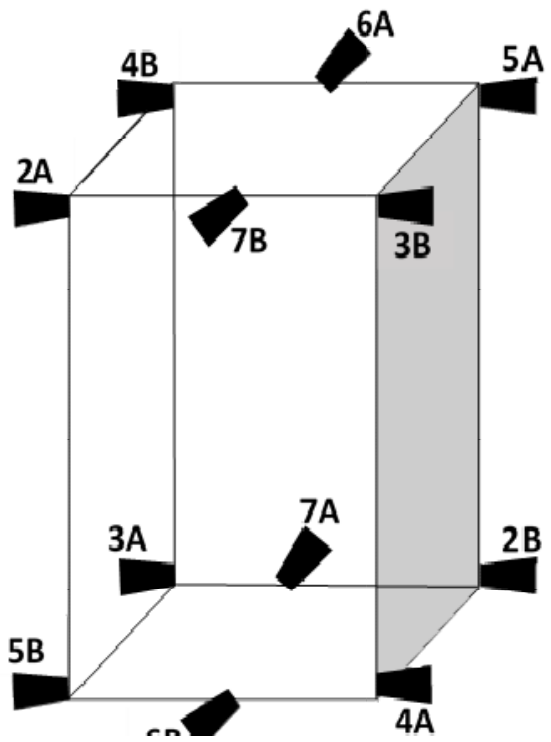

Fig.1 Thruster's configuration
The components of thruster force via the spacecraft three axis can be concluded from Figure 2 as follow [11]:

$$
\begin{gathered}
F_{\text {Thruster }}=F=10 N \\
F_{x}=F \cos \beta \cos \alpha \\
F_{y}=F \cos \beta \sin \alpha \\
F_{z}=F \sin \beta
\end{gathered}
$$

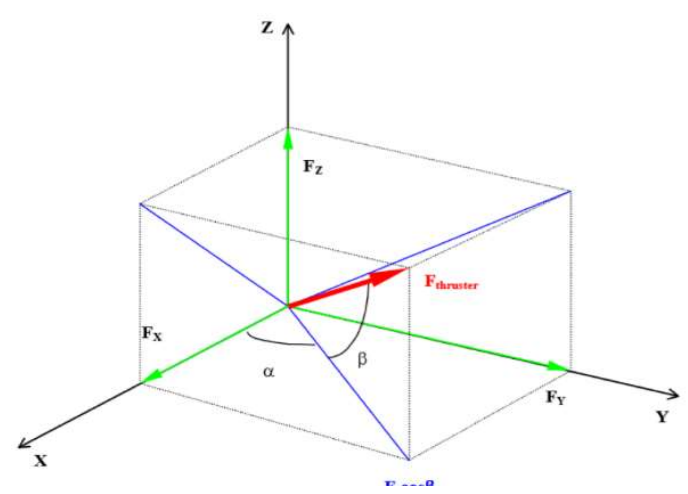

Fig.2 Thruster forces

\section{SATELLITE STATION KEEPING}

The most geostationary satellites are used for communication services due of the features of this kind of orbit, which permits the revolution with the same earth rotation period. However, the external perturbations can modify the satellite orbit, creating the orbit drift that can affect the coverage area and the signal quality as well as the mission services, therefore it is necessary to correct the orbit within specified limits and maintain the satellite in its desired position.

\subsection{Station keeping requirements}

Station keeping maneuver should be performed to maintain the satellite longitude and latitude around the nominal point within specified limits called control box (station keeping window) [1].

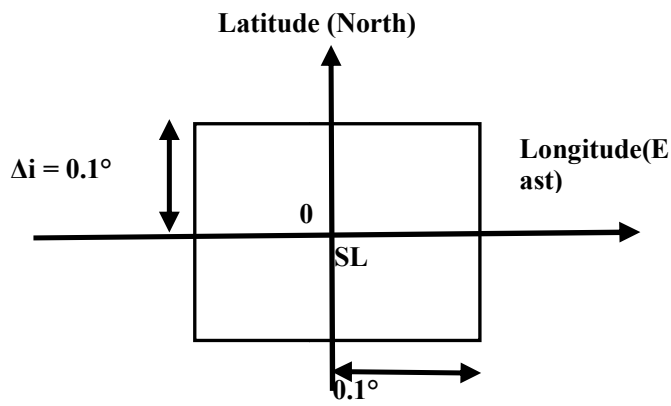

Fig. 3 Station keeping window Control box: longitude $\pm 0.1^{\circ}$, Latitude $\pm 0.1^{\circ}$ 
In station-keeping mode the attitude errors should be less than: Roll: $\pm 0.1^{\circ}$, Pitch: $\pm 0.1^{\circ}$, Yaw: $\pm 0.2^{\circ}$

After the transfer orbit the satellite will be located in its desired position, then the orbit control will consume the remaining propellant which be mainly used for station keeping maneuvers.

\subsection{Longitudinal drift}

EWSK is generally performed to correct two kinds of drift: the longitude drift caused by the Earth non-spherical perturbation and the eccentricity drift caused by the solar radiation pressure perturbation.

The EWSK consists of two kind of maneuvers westward control or eastward control. Under the natural perturbations, the satellite position (longitude) can define the direction of the satellite drift (Figure 4).

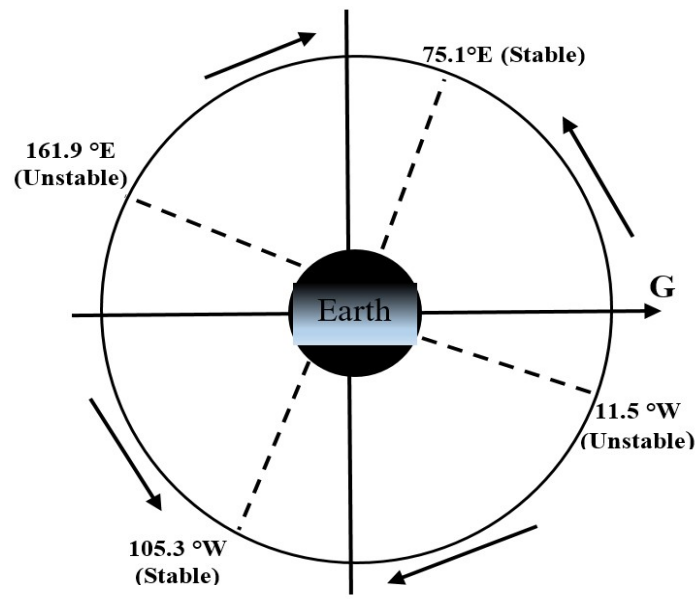

Fig.4 Longitudinal drift

In Figure 4 it can be observed that the orbit contains two stable positions and two unstable positions, the satellite will drift toward the near stable point, for example if a satellite in the position $20^{\circ} \mathrm{E}$ will drift toward the near stable point $75.1^{\circ} \mathrm{E}$, hence, eastward control will be needed.

\subsection{Station keeping strategy}

The aim of EWSK is to maintain the mean longitude in the drift interval $\left[\begin{array}{ll}\lambda_{0}-\Delta \lambda & \lambda_{0}+\Delta \lambda\end{array}\right]$

The EWSK can be designed as follow[1]:

Under the natural perturbations, the satellite moves taking the curve direction $A \rightarrow B \rightarrow C$. When the satellite reaches the curve west limit $C$ westward control will be applied to decrease the orbit semi-major axis that will change the satellite moving direction to be from $C \rightarrow A$ that is the EWSK period.

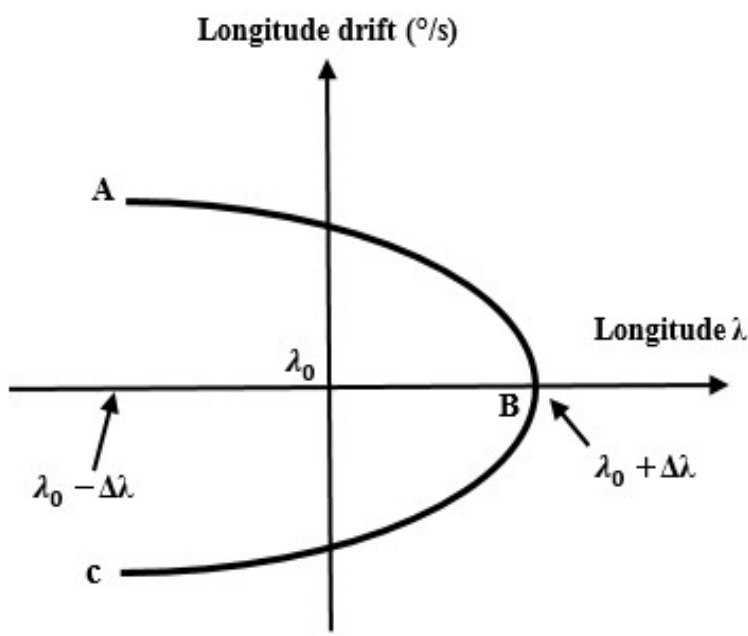

Fig.5 Station keeping strategy

\section{THRUSTER ATTITUDE CONTROL}

\subsection{Satellite dynamics}

The general satellite dynamics equation can be given as follow [12][13]:

$$
I \dot{\omega}+\omega \times(I \omega+h)=-\dot{h}+T
$$

Where $I$ is the satellite inertia matrix, $h$ is the angular momentum provided by the actuator, $\dot{h}$ is the control output.

The satellite attitude (orientation) in complex environments can be affected byaerodynamic torque $T_{d}$, gravity gradient torque $T_{g}$, geomagnetic torque $T_{m}$ and solar radiation pressure $T_{s r p}$.

The total environmental disturbance torque can be expressed as [12]:

$$
T=T_{d}+T_{g}+T_{m}+T_{s r p}
$$

$T=\left[\begin{array}{lll}T_{x} & T_{y} & T_{z}\end{array}\right]^{T}$ the external perturbation.

$h=\left[\begin{array}{lll}h_{x} & h_{y} & h_{z}\end{array}\right]^{T}$

$I=\operatorname{diag}\left(I_{x}, I_{y}, I_{z}\right)$

And the angular velocity with can be given by: 


$$
\omega=\left[\begin{array}{c}
\dot{\phi}-\omega_{0} \psi \\
\dot{\theta}-\omega_{0} \\
\dot{\psi}+\omega_{0} \phi
\end{array}\right]
$$

Where $\phi, \theta$ and $\psi$ are respectively the rollpitch and yaw satellite attitude angles and $\omega_{0}$ is the orbital velocity.

Finally the simplified dynamics equations of a rigid spacecraft can be expressed as follow [14]:

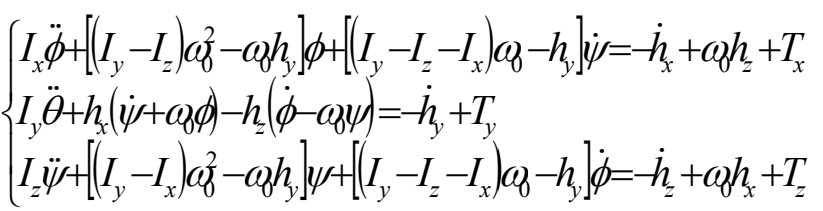

\subsection{Thruster model}

The actuators used in EWSK are thrusters, the satellite orbital position is $42^{\circ} \mathrm{E}$, hence, the satellite will drift in East direction (see Fig4).

Eastward force must be generated to correct this drift, according the thruster configuration in Figure 1. The thruster pair $2 \mathrm{~A}$ and $3 \mathrm{~A}$ (in nominal case, $4 \mathrm{~B}$ and $5 \mathrm{~B}$ in redundant case) will be used all along the orbital maneuver, however all six thrusters of branch A (nominal branch 2A-7A) will be used in the attitude control during all station keeping mode.

The Thrusters work in ON/OFF mode than their model can be presented in the figure below [14]:

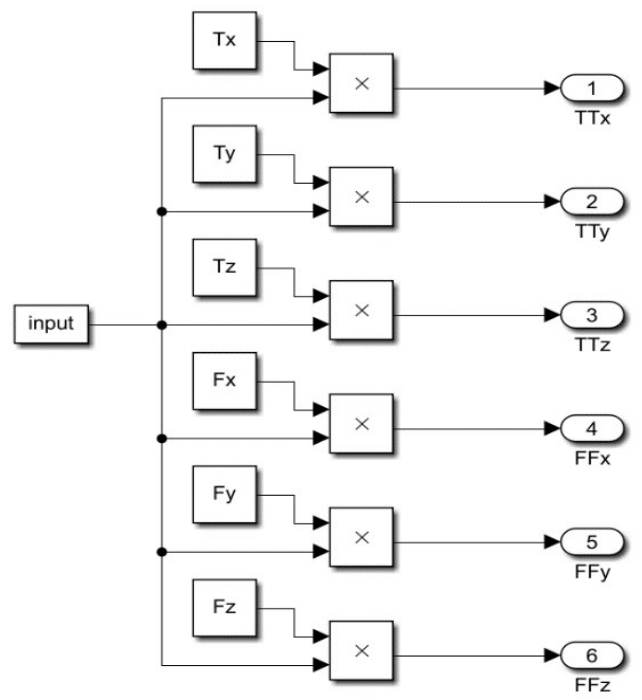

Fig.6 Thruster model

\subsection{Bang-Bang controller with dead-band}

For the attitude and orbit control using the thrusters as actuators, the appropriate control method is the PID controller, which provides a simple and reliable control solution respecting all mission requirements and performances. Furthermore, a Bang-Bang technique is associated to the classical PID controller to manage the ON/OFF thruster pulses[15].

Indeed, a dead-band is implemented in the simple Bang-Bang controller, which avoids the controller to generate output before the input reaches a certain threshold; this effect permits to minimize the switching frequency and the fuel consumption.

Hence, the proposed controller equation can be expressed as follow:

$$
u(t)= \begin{cases}U \operatorname{sign}(r(t)) & \text { if }|r(t)| \geq U_{O N} \\ 0 & \text { if }|r(t)| \prec U_{O N}\end{cases}
$$

The following figure illustrates the BangBang controller layout with dead-band:

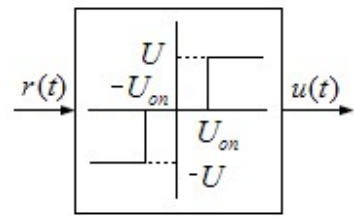

Fig.7 Bang-Bang controller with Dead-band

\section{NEMERICAL SIMULATIONS}

Numerical simulations have been performed using Matlab/Simulink environment in order to demonstrate the effectiveness of the proposed method. The obtained results use the parameters illustrated in Table 1.

Table 1 Satellite simulation parameters

\begin{tabular}{|l|c|}
\hline Parameter & Value \\
\hline Inertia matrix $\left[\mathrm{kg} . \mathrm{m}^{2}\right]$ & {$\left[\begin{array}{ccc}3770 & 0 & 0 \\
0 & 730 & 0 \\
0 & 0 & 4020\end{array}\right]$} \\
\hline Initial attitudes $\left[^{\circ}\right]$ & {$\left[\begin{array}{lll}0.1 & 0.2 & -0.03\end{array}\right]^{T}$} \\
\hline Initial angular rates $[\circ / \mathrm{s}]$ & {$\left[\begin{array}{lll}0 & 0 & 0\end{array}\right]^{T}$} \\
\hline Orbital velocity $[\mathrm{rad} / \mathrm{s}]$ & $7.2722 e-5$ \\
\hline
\end{tabular}




\begin{tabular}{|l|l|}
\hline External Torque $[N . m]$ & $\left\{\begin{array}{l}T_{x}=2.10^{-5}\left[1-\sin \left(\omega_{0} t\right)\right] \\
T_{y}=10^{-4} \cos \left(\omega_{0} t\right) \\
T_{z}=-5.10^{-5} \cos \left(\omega_{0} t\right)\end{array}\right.$ \\
\hline
\end{tabular}

The aim of the control is to maintain the desired attitude during the orbital maneuver (station keeping), the simulation results are shown in figure 8-13.

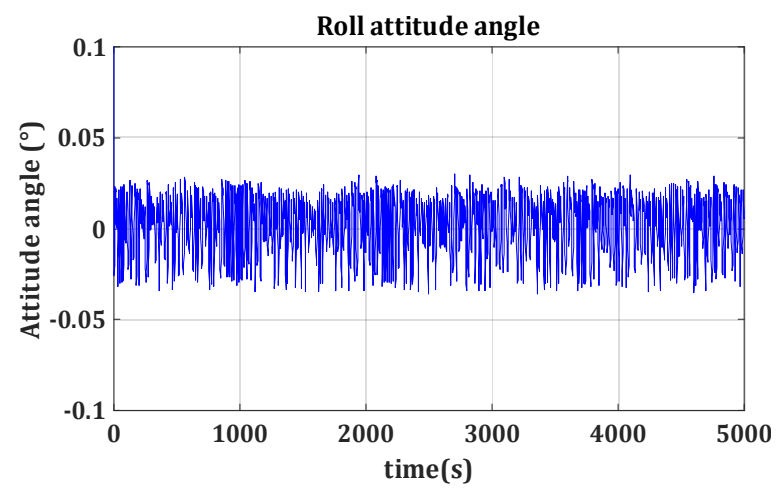

Fig.8 Roll attitude angle

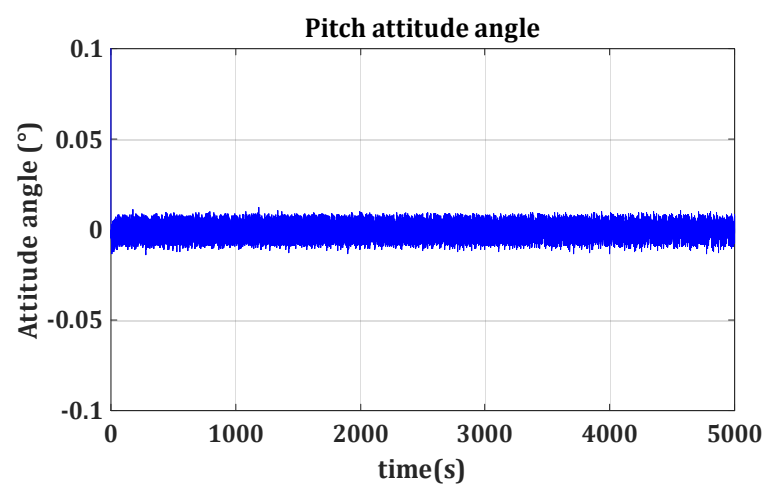

Fig.9 Pitch attitude angle

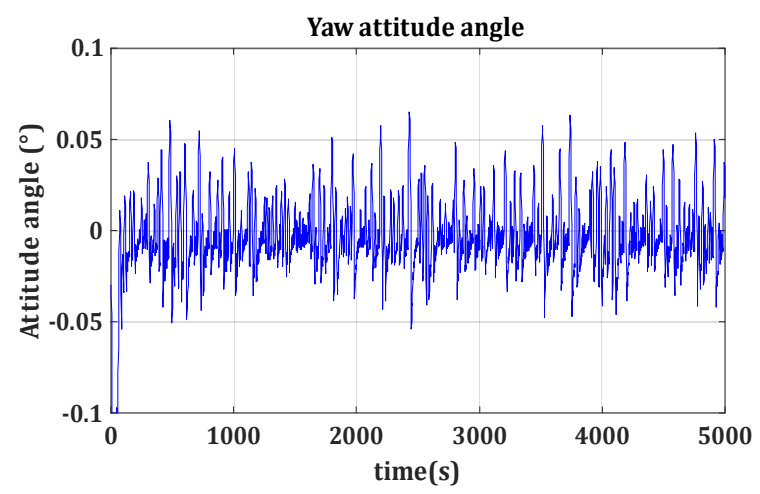

Fig.10 Yaw attitude angle
The time response of the attitude angles can be shown in figures 8-10, where the attitude accuracy has been reached, moreover, the errors have been reduced to the lowest, from this it can be noticed how the control system brings the satellite's attitude from the initial simulation values to $\pm 0.03^{\circ}$, $\pm 0.01^{\circ} \pm 0.06^{\circ}$ for roll, pitch and yaw attitude respectively, which meets the mission requirements $\left( \pm 0.1^{\circ}, \pm 0.1^{\circ}\right.$ and $\pm 0.2^{\circ}$ for roll, pitch and yaw, respectively). This result shows that the control system manages the system response in east/west station keeping.

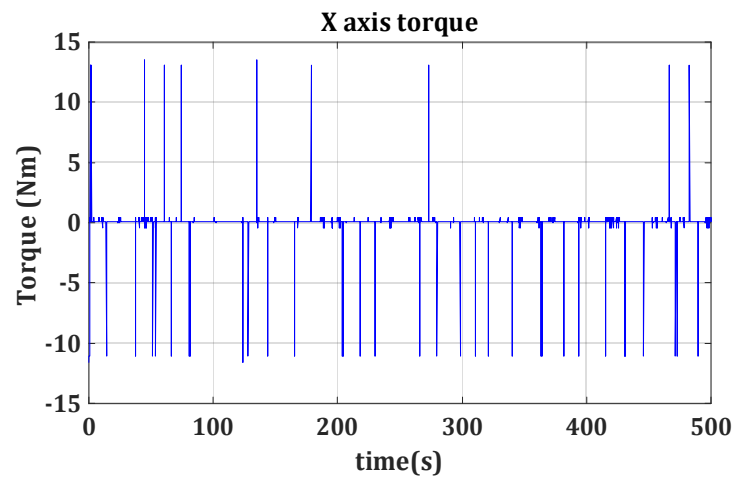

Fig.11 X-axis torque

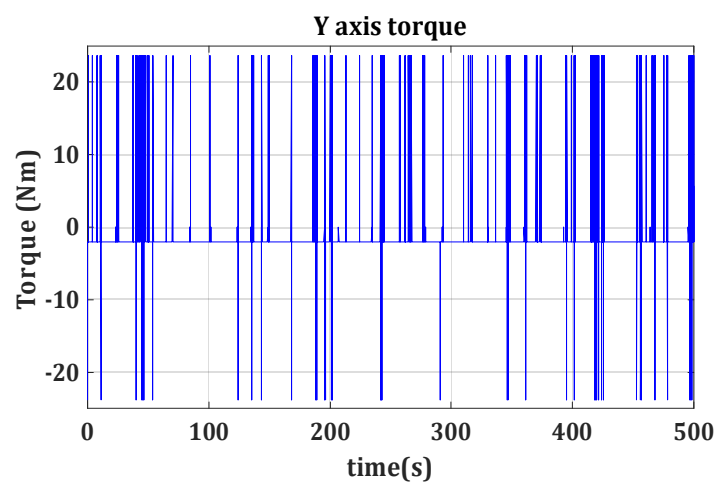

Fig.12 Y-axis torque

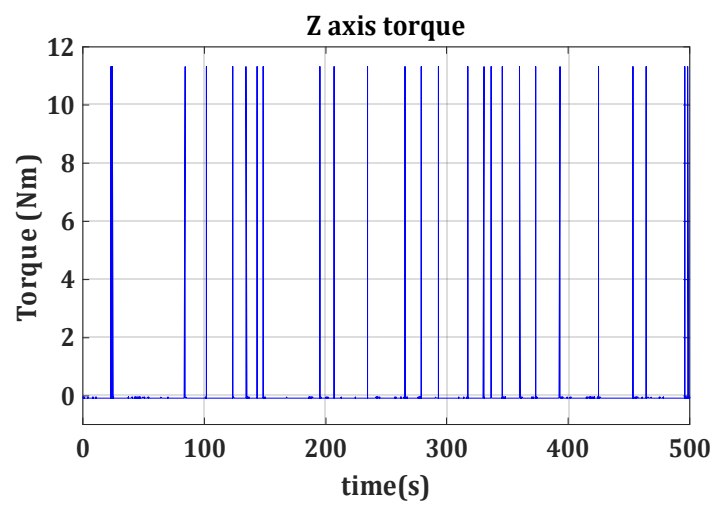

Fig.13 Z-axis torque 
Figures 11-13 represent the resulting thruster's actuations that accumulates the six thrusters generated torques in $\mathrm{x}, \mathrm{y}$ and $\mathrm{z}$-axis respectively. The generated actuations are managed by Bang-Bang controller with deadband technique for ON/OFF thruster's pulses, which permits to maintain the attitude control within the three axes, hence, respecting the pointing accuracy.

\section{CONCLUSION}

Attitude and orbit control during east west station keeping maneuver have been addressed using Bang-Bang techniquewith dead-band to manage ON/OFF thrusters pulses. The proposed strategy allows correcting the orbit drift without degrading the attitude control requirements as well as the pointing accuracy.

Numerical Simulations have been performed using MATLAB/SIMULINK environment. The simulation results show clearly the convergence of the attitude angles during the station-keeping maneuver, the controller can maintain perfectly the required pointing accuracy. Simulation results can also illustrate the feasibility and the effectiveness of the proposed methods that provide a useful and promising way for the attitude control of rigid spacecraft.

Some perspectives of this work can be also oriented toward the optimization of the controller gains and the fuel consumption, study of the case of electric or hybrid propulsion, development of other modulations techniques with better performance, design another technique which takes into account inertia uncertainties problem.

\section{References}

[1] Borissov, Stoian, Yunhe $\mathrm{Wu}$, and Daniele Mortari. "East-west GEO satellite stationkeeping with degraded thruster response." Aerospace 2.4 (2015): 581-601.

[2] Losa, D., Lovera, M., Marmorat, J. P. Dargent, T., \& Amalric, J. (2006, October). "Station keeping of geostationary satellites with on-off electric thrusters". In 2006 IEEE Conference on Computer Aided Control System Design, 2006 IEEE International Conference on Control Applications, 2006
IEEE International Symposium on Intelligent Control (pp. 2890-2895). IEEE.

[3] Romero, P., Gambi, J. M., Patiño, E., et al. "Optimal station keeping for geostationary satellites with electric propulsion systems under -eclipse constraints". In Progress in Industrial Mathematics at ECMI 2006. Springer, Berlin, Heidelberg, 2008. p. 260264.

[4] G. Campan, F. Alby, and H. Gautier, "Les techniques de maintien a' poste de satellites geostationnaires", in ' Mecanique spatiale' , CNES, Ed. Toulouse, France: Cepadu esEditions, 1995, ch. 15, pp. 983-1085.

[5] M. Sidi, "Spacecraft Dynamics and Control". Cambridge University Press, 1997.

[6] Kienitz, K. H., "Attitude stabilization with actuators subject to switching restrictions: an approach via exact relay control methods," IEEE Transactions on Aerospace and Electronic Systems, Vol. 42, No. 4, 2006, pp. 1485-1492.

[7] Brown, T., "In-flight tuning of the Cassini RCS attitude controller," AIAA Guidance, Navigation, and Control Conference, Portland, Oregon, 2011.

[8] Jeon, S. W. and Jung, S., "Hardware-in-theloop simulation for the reaction control system using PWM-based limit cycle analysis," IEEE Transactions on Control Systems Technology, Vol. 20, No. 2, 2012, pp. 538545

[9] Agrawal, B. N. and Bang, H., "Robust ClosedLoop Control Design for Spacecraft Slew Maneuver Using Thrusters," Journal of Guidance, Control, and Dynamics, Vol. 18, No. 6,1995 , pp. 1336-1344.

[10] Burdick, G. M., Lin, H.-S., and Wong, E. C., "A Scheme for Target Tracking and Pointing During Small Celestial Body Encounters," Journal of Guidance, Control, and Dynamics, Vol. 7, No. 4, 1984, pp. 450-457.

[11] Derman, Hakki Özgür. "3-Axis attitude control of a geostationary satellite". Diss. MSc Thesis, Turkey, Middle East Technical University, 1999.

[12] Benmansour, J. E., Benfriha, E., \& Roubache, R. "Satellite Attitude Control Based Adaptive sliding Mode Method", Algerian Journal of Engineering Research, Volume 4, Numéro 1, Pages 6-11, 2019.

[13] Benmansour, J. E., Khouane, B., \& Rima, R. (2018). "High Precision Roll/Yaw Attitude Stabilization for Flexible communication satellite". Algerian Journal of Signals and Systems, 3(2), 81-88.

[14] Salomón, Montenegro, and Amézquita Kendrick. "Accomplishing Station Keeping Mode for Attitude Orbit Control Subsystem Designed for T-SAT." The Sixth International Symposium on Neural Networks (ISNN 2009). Springer, Berlin, Heidelberg, 2009.

[15] Bekir, Esmat. "A tutorial on bang-bang algorithm for attitude control system". Usak University Journal of Engineering Sciences, 2018, vol. 1, no 2, p. 56-65. 